\title{
ON RADIO INTERFERENCE FILTER MEASUREMENT
}

\author{
Karel Hoffmann and Zbynek Skvor \\ Czech Technical University in Prague, Dpt. Electromagnetic Field, \\ Technicka 2, 16627 Praha 6, Czech Republic, voice: $+420-224352$ 276, \\ e-mail: hoffmann@fel.cvut.cz, skvor@fel.cvut.cz
}

\begin{abstract}
A new measurement method for on site testing of multiport radio interference filters (RIF) on shielded chamber is designed. The method is based on scalar measurement only and makes possible a pessimistic estimate of the filters damping in frequency band from DC - $3 \mathrm{GHz}$. More than $120 \mathrm{~dB}$ dynamic of measurement can be expected. At the time being there is no acceptable standard for these multiport measurements.
\end{abstract}

\section{INTRODUCTION}

Modern communication devices as well as clock frequencies of desktop computers are climbing well into gigahertz region. Preventing electromagnetic interference and/or information leakage gets more and more important and difficult at the same time.

Some premises handle data so important that there is a strong need for data protection against leakage caused by radiofrequency leakage. Such premises are often shielded and all lines connecting these premises with the rest of world should be safeguarded against leakage. This applies not only to data transmission lines, but also to power network lines. These lines are blocked using radiofrequency filters. Such filters need to be measured and periodically tested onsite.

Measurement of these devices is specific in several ways:

- In order to ascertain data protection, attenuation over $120 \mathrm{~dB}$ is required.

- At the time being, it is desired to test such devices from DC up to $3 \mathrm{GHz}$.
- The device itself is designed to handle and withstand substantial voltages and currents, resulting in big dimensions (up to one meter), and is equipped by big nuts and bolts, which are quite different from common precise microwave connectors.

Some basic information concerning the measurement of interference filters can be found in standard [1]. It uses scalar measurements only. The access is based on classic circuit theory and therefore the recommendations are useful at low frequencies only, being of a little use at higher frequencies of $\mathrm{GHz}$ region. The standard [1] is focused namely on two-conductor filters (one phase and Neutral in a grounded metal box). Only some general recommendations can be found with respect to measurement of multiport filters. Questions important on high frequencies concerning reference planes of measurement, calibration of measurement system, mode conversion and even measured data evaluation with respect to demanded insertion loss are not referred to.

This paper attempts to give a critical evaluation of the standard [1] and to design a new measurement method valid in frequency band up to $3 \mathrm{GHz}$ based on scalar measurements only.

Problems concerning three phase RIF filters are discussed. Conclusions are valid also for one phase RFI filters after a corresponding simplification.

\section{THEORETICAL BACKGROUND}

A typical RIF filter (Fig. 1.) has got four inputs and four outputs (three phase voltages L1, L2, L3 and Neutral N) and is placed in a grounded 
metal case, see Fig.1. The case contains also input and output metal boxes, not depicted in the figure, where the power cables are fixed on the terminals. The case of the filter is usually fixed on the wall outside the shealded chamber. The output power cable gets into the mounting box directly via a proper hole through the chamber wall, which insures its good shielding.

From microwave point of view this is an 8-port. Such a structure supports at least four modes of signal propagation. Information leakage can occur if any of these modes is not damped properly. It would be ideal to feed the input by these four mode voltage waves and measure the outgoing wave at the other side of the filter. Unfortunately the modal voltages are unknown. It would be possible to obtain these modes after complete scattering parameters were measured. Scalar measurement does not enable for mode determination.

Four modal voltage waves create an unknown base in a 4 dimensional space of the signal propagation in the filter. Modal voltage waves vary along the filter. The simplest but still unknown structure can be expected on the input and output power cables. Fortunately the space of signal propagation can be mapped over, not only by the modal waves but also by any orthogonal or orthonormal base of the space. From practical point of view it seems to be the best choise to use four modes where the ground

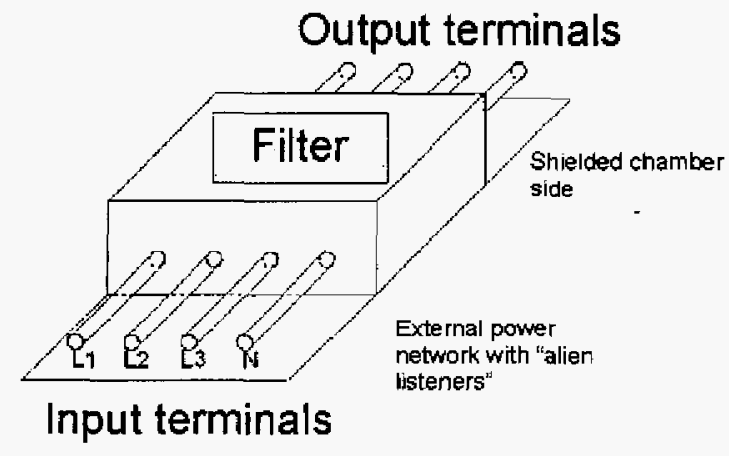

Fig. 1. An example of a RFI filter.

wire is a common reference and individual "active" wires L1, L2, L3, N are for individual modes excited step by step by incident voltage wave leaving remaining wires with no incident signal. It means that four modes with amplitudes $(1,0,0,0),(0,1,0,0),(0,0,1,0)$ and $(0,0,0,1)$ of incident waves on "active" wires can be supposed. Inside the filter any mode conversion must be supposed, see [2] for four-port case, and therefore a transmission from single input port to all output ports must be measured. It results in 16 transmission scalar measurements for the filter, with $16\left|s_{i j}\right|$ parameters obtained.

The most important question in the RIF filter testing is the definition of its insertion loss. With respect to practical applications the test should insure that the filter allows no signal propagation with smaller attenuation than certain desired value. We suggest that the quantity for the filter attenuation should be defined for a multimode excitation as a minimum of the ratio of incident input power to output passed thru power

$$
B=\min \frac{P_{i n}}{P_{o u t}}=\min \frac{\sum_{i=1}^{4} P_{i n, i}}{\sum_{i=5}^{8} P_{o u t, i}}
$$

Where B stands for the value of damping, $P_{\text {out } i}$ stands for the power carried by the wave leaving i-th port at the output side and $P_{i n, i}$ stands for the power carried by the wave incident to $i$-th port at the input side of the filter. Summations are carried over 4 terminals, at input or output.

The value of $P_{\text {in }} / P_{\text {out }}$ in (1) varies in multimode excitation of the filter with respect to the distribution of the input power into individual modes and phase shifts of individual incident voltage waves. The physical reason is certain mutual compensation of outgoing waves on the input ports.

The worst case given by (1) can be exactly determined if the vector scattering parameters of the filter were known. The process can be carried out so that the amplitude and phase distribution of signals fed into the input terminals is optimized for maximum pover delivered to loads at output terminals. 


\section{NEW APPROACH}

Scalar measurement gives only partial information about the filter properties. Therefore only some worst case estimation of damping $B$ given by (I) can be done.

The superposition principle demands that the (output) voltage caused by several input voltages can be obtained by a simple addition of output voltages caused by single input sources. As the transmissions are complex numbers, some of the partial output voltages are out of phase and cancel each other. If only magnitudes are known, the worst-case estimation of output voltages is obtained as a sum of magnitudes. Therefore the worst case estimation of $B$ can be expressed as:

$$
B=\min \frac{1}{\left.\sum_{i=1}^{4}\left(\sum_{j=5}^{8} a_{i} \mid s_{j, i}\right)\right)^{2}}
$$

Where $\left|s_{i, j}\right|$ are measured amplitudes of scattering transmission coefficients of the filter, $i$ stands for the input ports and $j$ for the output ports and input voltage waves $a_{i}$ are normalized to unity input power, eg.

$$
\sum_{i=1}^{4}\left|a_{i}\right|^{2}=1
$$

Some explanation to eq. (2) can be given:

Filter attenuation (1) depends on phase and amplitude distribution of input signal at ports 1 to 4. Allowing output signals to add in phase means that the worst possible case is taken into account with respect to all possible phases of input signals as well as (unknown) phases of scattering parameters of the filter. When phases fixed the lowest possible attenuation occures for a certain combination of input voltage wave amplitudes. This combination can be found by optimization. The optimization finds the values of input waves $a_{1}, \ldots a_{4}$ constrained by nonmalization (3) minimizing the damping $B$ given by (2).

\section{PRACTICAL RECOMMENDATIONS}

\section{A. Vector and Scalar Measurement}

The vector measurement makes possible to determine scattering parameters of the 8-port of the filter. They include complete if. information about the properties of the filter. Applying these parameters in a proper microwave circuit simulator the behavior of the filter for different impedances connected to the terminals can be analyzed and predicted for practical applications.

From this point of view the vector measurement is the best choice. From practical point of view the scalar measurement if often preferred to in the case of on-site measurements. Usually the generator is outside and the spectrum analyzer inside the closed shielded chamber. This arrangement minimizes cross talks due to leakage of the measurement cables and covers of the mounting boxes. It makes possible to achieve high dynamic measurement with reasonable instrument cost.

Unfortunately it gives only partial information. This measurenent does not yield enough information for prediction of filter behavior when connected to impedances different from the impedance of the measurement instruments. Therefore the standard [1] recommendation to measure the filter also with significantly different impedances connected to the input and output ports is a reasonable choice.

\section{B. Reference Planes and System Impedances}

The standard [1] does not deal with this question. It demands to measure the filter in $50 \Omega$ measurement system by the standard method. Also recommended approximation method needs to measure in a measurement system with impedance $0.1 \Omega$ on the input and $100 \Omega$ on the output of the filter and vice versa. With respect to dimensions it is not possible to connect $0.1 \Omega$ line to terminals separated of several centimeters apart from the realization problem of such a line. 
Reasonable solution seems to be to put the reference planes on some hole of the walls of the mounting boxes. It corresponds to reference planes placed on the input and output power cables. The hole from the output mounting box to the shielded chamber is the best choice for the output. In the input the hole for the input cable can be used as well or an additional hole can be used in the box cover. This choice of reference planes is advantageous with respect to ground connection of corresponding coaxial connectors. Moreover the measurement approximates conditions of real applications of the filter with respect to wire arrangement in the mounting boxes.

The demand of $0.1 \Omega$ for the approximation method measurement is rather unpractical with respect either to realization of such a line or an attenuation of the corresponding impedance transformer. The value of $1 \Omega$ seems to be a reasonable compromise.

\section{Calibration of Measurement System}

The standard [1] is based on voltage measurements valid on low frequencies. It recommends carrying on the insertion loss calibration by direct connection of input and outputting measurement ports. In microwave frequency region good matching for scalar calibration is a must. Therefore the recommended calibration cannot be used. In the case of approximation method, see [1], the calibration for measurement with different impedances on the input and output of the filter can be done by the following way. The measurement needs to use impedance transformers from impedances of the instruments to impedances $0.1 \Omega(1 \Omega)$ and $100 \Omega$. The calibration should be carried on therefore without these transformers. Their insertion loss must be subtracted from measured values subsequently.

\section{Measurement Dynamic}

A frequency wide-band measurement with $0,1 \Omega$ and $100 \Omega$ impedances of measurement ports needs to use resistive impedance tranformers. Their insertion losses when connected into $50 \Omega$
$-1 \Omega$ and $50 \Omega-100 \Omega$ measurement systems are ideally $23 \mathrm{~dB}$ and $7 \mathrm{~dB}$. It means that the measurement system dynamic is decreased of $30 \mathrm{~dB}$. A reasonable input power with respect to power consumption in the $50 \Omega-1 \Omega$ impedance transformer is $26 \mathrm{dBm}$. It results in $120 \mathrm{~dB}$ dynamic of measurement when a spectrum analyzer with noise floor below $13 \mathrm{JdBm}$ is used.

\section{MEASUREMENT METHOD}

Taking into account the above mentioned theoretical background and practical recommendation a new on site scalar measurement method for RIF filters based on [1] but modified for microwave frequencies can be briefly formulated.

1. Measure the tested filter with three different impedances on test ports of measurement system: $.50 \Omega-50 \Omega, 1 \Omega-$ $100 \Omega$ and $100 \Omega-1 \Omega$.

2. Measure transmissions from all input ports to all output ports. Consider recommendations with respect to reference planes positions and calibration.

3. Determine the filter attenuation using (2) with attenuation of impedance transformers considered.

\section{CONCLUSION}

A new measurement method for on site testing of multiport radio interference filters on shielded chamber was designed. The method extends limits of European standard CISPR 17: 1981 from one-phase filters towards multiport multiphase filters and towards microwave frequencies. The method has no upper frequency limit in principle.

\section{ACKNOWLEDGMENT}

This research has been supported by grant no. 2002200212 of the National Security Authority of the Czech Republic as well as research program J04/98: 210000015 and grant No. 102/01/0573 of Czech Grant Agency. 


\section{REFERENCES}

\section{[1] European standard CISPR17: 1981}

[2] Bockleman D. E. and Eisenstadt W. R.: Combined Differential and Common-Mode Scattering Parameters: Theory and Simulation. IEEE Trans. On Microwave Theory and Techniques, vol. MTT-43, No. 7., July 1973, pp. 450-457 